\title{
Midterm Results After Subtrochanteric End-to-Side Valgization Osteotomy in Severe Infantile Coxa Vara
}

\author{
Christian M.J. Günther, MD, ${ }^{*}$ Moritz Komm, MD, $\dagger$ Volkmar Jansson, MD, PhD,* \\ and Bernhard Heimkes, MD, PhD*
}

\begin{abstract}
Background: For the treatment of the severe infantile coxa vara it is mandatory for the orthopaedic surgeon to observe the mechanobiology of the growing hip before and after the surgical intervention. We hereby would like to present our experiences with the subtrochanteric end-to-side valgization osteotomy and to compare the procedure with the alternatively used Y-shaped osteotomy as described by Pauwels.
\end{abstract}

Methods: Thirteen patients (20 hips) who had undergone subtrochanteric end-to-side valgization were followed for a mean 6.2 years (range, 0.8 to $12.8 \mathrm{y}$ ). At the time of surgery the mean age was 7.1 years (range, 2.0 to $13.3 \mathrm{y}$ ), last follow-up examination was performed at a mean of 13.4 years of age (range, 5.1 to $18.3 \mathrm{y}$ ). The deformities were etiologically based on 5 entities: congenital coxa vara $(n=1)$, osteochondrodysplasias $(n=12)$, postosteomyelitic coxa vara $(n=5)$, and avascular femoral head necrosis in the course of congenital dysplasia of the hip $(n=2)$. The follow-up rate was $100 \%$. In addition, we analyzed a total of 93 pelvic radiographies with a total of 139 hip joints. Thirty angles and distances were assessed according to parameters described in the literature.

Results: Although preoperatively 12 patients presented with a positive Trendelenburg's sign, it was only present postoperatively in 2 patients. Duchenne's limp reduced from 10 to 1 . All of the 15 preoperatively apparent nonunions could be healed by means of surgery. Two hips redeveloped pathologically lowered collodiaphyseal angles postoperatively, one of which had to undergo revision surgery. Preoperatively 15 out of 20 patients $(75 \%)$ showed nonunions all of which healed after surgery. No recurrence could be seen at the time of the last follow-up. The following angles were assessed on plain radiographies of the pelvis preoperatively and directly postoperatively as well as on the last follow-up at a mean of 85 months: CCDangle 98 degrees/156 degrees/144 degrees, EY-angle 55 degrees $/ 5$ degrees/15.7 degrees, AY-angle 32 degrees/75 degrees/66 de-

From the *Department of Orthopaedic Surgery, University Hospital of Munich (LMU) Campus; and †Department of General, Visceral, Thoracic and Vascular Surgery, Munich Municipal Hospital, Munich, Germany.

C.M.J.G. and M.K. contributed equally.

None of the authors received financial support for this study.

The authors declare no conflict of interest.

Reprints: Bernhard Heimkes, MD, PhD, Department of Orthopaedic Surgery, University Hospital of Munich (LMU) Campus, Grosshadern Marchioninistr. 15 81377, Munich, Germany. E-mail: bernhard. heimkes@med.uni-muenchen.de.

Copyright (C) 2013 by Lippincott Williams \& Wilkins grees, CE-angle 20 degrees/25 degrees/18 degrees, AC-angle 20 degrees/18 degrees/20 degrees. The articulotrochanteric distance was $5 \mathrm{~mm} / 26 \mathrm{~mm} / 14 \mathrm{~mm}$.

Conclusions: The subtrochanteric end-to-side valgization osteotomy showed to be highly effective in the management of the infantile coxa vara, improving the clinical impairment of the patients postoperatively. All of the preoperatively present nonunions showed osseous consolidation at follow-up examination. Only minor revarization tendencies could be found. The procedure is technically less demanding, safer and more efficient regarding the lengthening of the affected limb in comparison to the Y-shaped intertrochanteric osteotomy as described by Pauwels.

Level of Evidence: Case-control study (EBM-level III).

Key Words: infantile coxa vara, developmental coxa vara, spondylo-epiphyseal dysplasia, femoral neck pseudarthrosis, subtrochanteric end-to-side valgization osteotomy

( $J$ Pediatr Orthop 2013;33:353-360)

$V^{2}$ arus deformities of the infantile hip are encountered very infrequently. Thus, it is not surprising that only few studies on their management can be found in the international literature. Most of these studies include a small number of cases ${ }^{1-6}$ and only 4 studies comprise 20 or more cases. ${ }^{7-10}$

As the few cases that have been written up in the international journals are caused by a multitude of underlying diseases, it is also not astonishing that no broadly accepted classification exists. Therefore, we would like to propose the following:

1. Congenital coxa vara (synonyms: coxa vara infantum, idiopathic coxa vara, primary coxa vara, developmental coxa vara).

2. Coxa vara in the course of spondylo-epi-metaphyseal dysplasias.

3. Secondary coxa vara caused by locally deforming hip diseases (avascular necrosis after congenital hip dysplasia, osteomyelitis/coxitis, Legg-Calvé-Perthes disease, trauma, tuberculosis, benign tumors or tumor-like lesions, fibrous dysplasia of bone).

4. Coxa vara in systemic osteopenia (rickets, osteogenesis imperfecta, meningomyelocele).

Pathogenetically a coxa vara mostly emerges out of a biological insufficiency of the femoral neck or a disturbance of the epiphyseal growth plate, both of which 


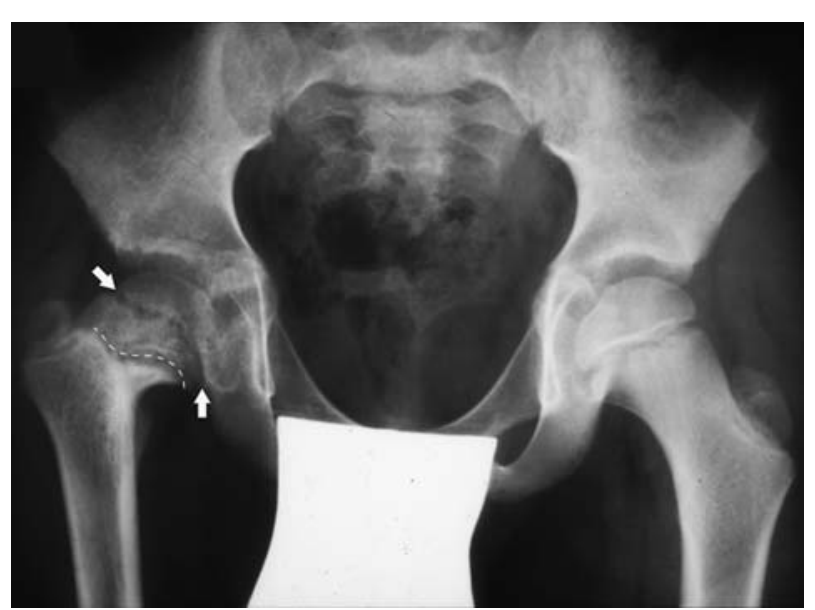

FIGURE 1. Unilateral coxa vara of the right side with a perpendicular epiphyseal plate (arrows), horizontal apophyseal plate and nonunion of the femoral neck (dotted line).

are incapable of handling force loads of everyday activities. This results in a decreasing angle between the femoral head's center, the femoral neck and the diaphysis [caput collum diaphyseal (CCD) angle]. Simultaneously, a verticalization of the epiphyseal plate and a cranialization of the greater trochanter occur, leading to pathogenetic bending and shear loads of the femoral neck ${ }^{11,12}$ and ultimately a crook deformity coxa vara (Fig. 1).

The varization of the femoral neck and the consequent overgrowth of the greater trochanter bring the hip abductors' sites of origin and insertion closer together, which results in decreasing tension and thus efficiency. Clinically this shows in a positive Trendelenburg's sign, Duchenne's limp, ${ }^{12,13}$ and shortening of the affected leg. Amstutz and Philip ${ }^{14}$ also described an insufficiency of the gluteus maximus muscle to erect the pelvis, resulting in considerable lordosis of the lumbar spine. Considering these changes, the therapeutic goals consist of the morphogenic overcorrection of the deformity leading to a transduction of the neck's shear into compressive forces, equalization of the limbs' length differences, and the normalization of the hip abductors' and the gluteus maximus's initial tension.

Pauwels presented the underlying principles in his hip model established in 1935. He described the biomechanics of the hip and the forces taking effect at the coxal end of the femur, with special emphasis on the resulting hip force vector R. ${ }^{15}$

Heimkes et $\mathrm{al}^{16}$ and later on Skuban et $\mathrm{al}^{17}$ refined Pauwel's hip model, establishing the Clustered Pillar Concept in which they underline the influence of the trochanteric apophyseal growth on the modelling of the coxal femur. Realizing that the trochanter is under pressure from a force vector originating laterocranially, which itself is the functional result of an anatomical doublelayer muscular tension banding, they constructed the trochanteric resulting vector $\mathrm{R}_{\mathrm{t}}$. Its direction and absolute value arise from a force parallelogram, composed of the muscular resultant $F_{m t}$ (which represents the forces affecting the trochanter cranially), the vector $F_{m k}$ and the caudally effecting forces. In combination Heimkes concluded, $\mathrm{R}_{\mathrm{h}}$ and $\mathrm{R}_{\mathrm{t}}$ define the collodiaphyseal angle (Figs. 2A-C).

As displayed in the hip model (Figs. 2A-C), a diminished neck-shaft angle results in bending loads to the femoral neck characterized by the hip joint resultant $R_{h}$ as well as the verticalization of $R_{t}$, which is caused by weakened hip abductors in the presence of physiologically working knee extensors. This causes the trochanteric apophysis to grow in a cranial direction, bringing the abductors' origins and insertions closer together, thus lowering their efficiency. As a result, the vector $F_{m h}$ continues to shorten, further steepening $\mathrm{R}_{\mathrm{t}}$. This downward spiral can only be stopped by restoring the normal tension load of the hip's abductors through distal relocation of the greater trochanter.

In the past, the most common method to achieve this objective, was the lateral extraction of an intertrochanteric wedge as described by Pauwels, ${ }^{18}$ which leads to an increased collodiaphyseal angle. As early as 1966 , Borden et $\mathrm{al}^{19}$ presented a subtrochanteric valgizating osteotomy, which is outlined below.

Surgery is recommended for hips with neck-shaft angles below $110^{\circ}$. It can also be done in coxa vara with osteochondrodysplasia, provided a nearly physiological joint geometry exists, consisting of a round femoral head, a physiologically shaped acetabulum and sufficient containment of the head. Further indications include secondary coxa vara after femoral head necrosis, LeggCalvé-Perthes' disease, osteomyelitis, bacterial hip arthritis, tumor, or trauma. For all of these conditions, nearly normal joint geometry is a prerequisite for surgery. In any case where a lateral misalignment of the femoral head following the valgization is suspected, this should be excluded by intraoperative arthrography before beginning the procedure.

Because there is a chance of spontaneous normalization in infants, the procedure is rarely performed before the age of 4 . In children older than 11, the operation leads to a significant increase in soft tissue tension, making the shortening intertrochanteric osteotomy the first choice for adolescents.

The procedure is not recommended for patients with a locally or systemically compromised bone metabolism, such as osteogenesis imperfecta, rickets, or fibrous dysplasia of bone.

\section{METHODS}

\section{Clinical Data}

Thirteen patients (6 male, 7 female) with 20 hips (9 male, 11 female) who underwent surgery between 1990 and 2004 were included in the study. Seven children had bilateral deformities and were treated on both sides. The diseases underlying the varus hips were congenital coxa vara $(n=1)$, osteochondrodysplasia $(n=12)$, osteomyelitis/bacterial hip arthritis $(n=5)$, and necrosis of the 

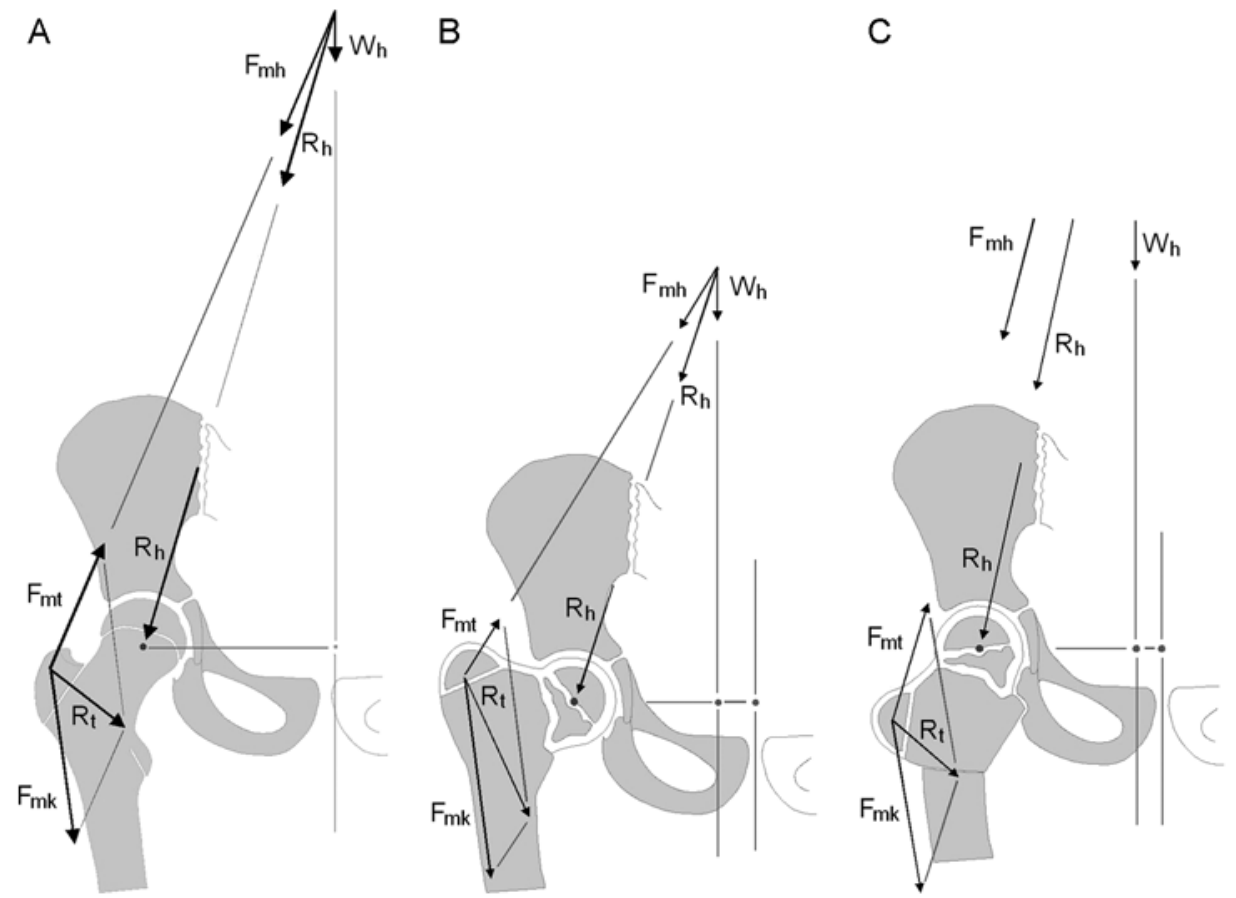

FIGURE 2. A, The development of the normal hip joint evolves out of the hip joint's resulting force vector $R_{h}$ and the trochanteric resultant force vector $R_{t}$. B, Due to the weakened and oblique character of the hip abducting musculature in coxa vara, the hip joint resulting force $R_{h}$ generates bending forces in the area of the femoral neck. Since, at the same time, the knee extending muscles work in a normal manner, the trochanteric resultant $\mathrm{R}_{\mathrm{t}}$ orientates perpendicularly, causing the trochanteric apophysis to grow cranialwards (greater trochanteric rocket). This leads to an increasingly elevated position of the trochanter and in last consequence to a crook-shaped coxa vara. $C$, By means of the end-to-side valgization procedure both the resultants $R_{h}$ and $R_{t}$ are virtually normalized regarding direction and absolute value. Thus, the femoral neck's nonunion is enabled to heal while the trochanteric growth is redirected craniolaterally.

femoral head after congenital displacement of the hip $(\mathrm{n}=2)$.

At the time of surgery, the mean age was 7.1 (range, 2.0 to 13.3 ) years, mean age at the time of the last followup examination was 13.4 (5.1 to 18.3$)$ years yielding a mean follow-up of $6.2(0.8$ to 12.8$)$ years. One boy was operated upon twice due to a recurring deformity on one of his hip joints, both of which had been treated. Since case numbers are based on hip joints rather than procedures, the double procedure is outlined in the respective area. All patients who received surgery could be examined clinically and radiologically, leading to a follow-up rate of $100 \%$.

Clinical parameters assessed were difference of leg length (LLD), Trendelenburg's sign, and Duchenne's limp. Leg length data were acquired by measuring the distance of the anterior superior iliac spine to the medial malleolus. Trendelenburg's sign was considered positive, if the patient's pelvis sagged toward the unsupported side when standing on the ipsilateral leg that had been operated upon. The presence of a Duchenne limp was noted, when the upper part of the body could not be stabilized during gait, resulting in a rocking motion towards the postsurgical side.

\section{Surgical Procedure}

Before the surgery, pelvis and long-leg x-rays have to be performed to allow for preoperative planning, which aims at a parallel orientation of the epiphysial plate and Hilgenrainer's line. This usually requires a blade plate with an angulation of 140 to 170 degrees, depending on the severity of the deformity. After standard lateral approach to the proximal femur, a guide wire is inserted as planned followed by a cannulated chisel. Both instruments are removed and the chosen blade plate is inserted into the preformed slot. Rotation has to be marked using $2 \mathrm{~K}$-wires above and beneath the designated osteotomy plane. The cut is performed horizontally in anteriorposterior direction one shaft width distal to the insertion point of the plate. Subsequently the proximal fragment is tipped laterally and reduced end-to-side. Then the angled plate is fixed to the femoral shaft using standard cortical screws. Finally, the position of the osteosynthesis is controlled by fluoroscopy and the wound is closed. For a detailed description of the technical procedure, please refer to the article published by Heimkes et a ${ }^{20}$ (Figs. 3A, B; Figs. 4A, B; Figs. 5A-C).

Postoperatively, in children below the age of 7 , we recommend spica casting, although the age threshold may 

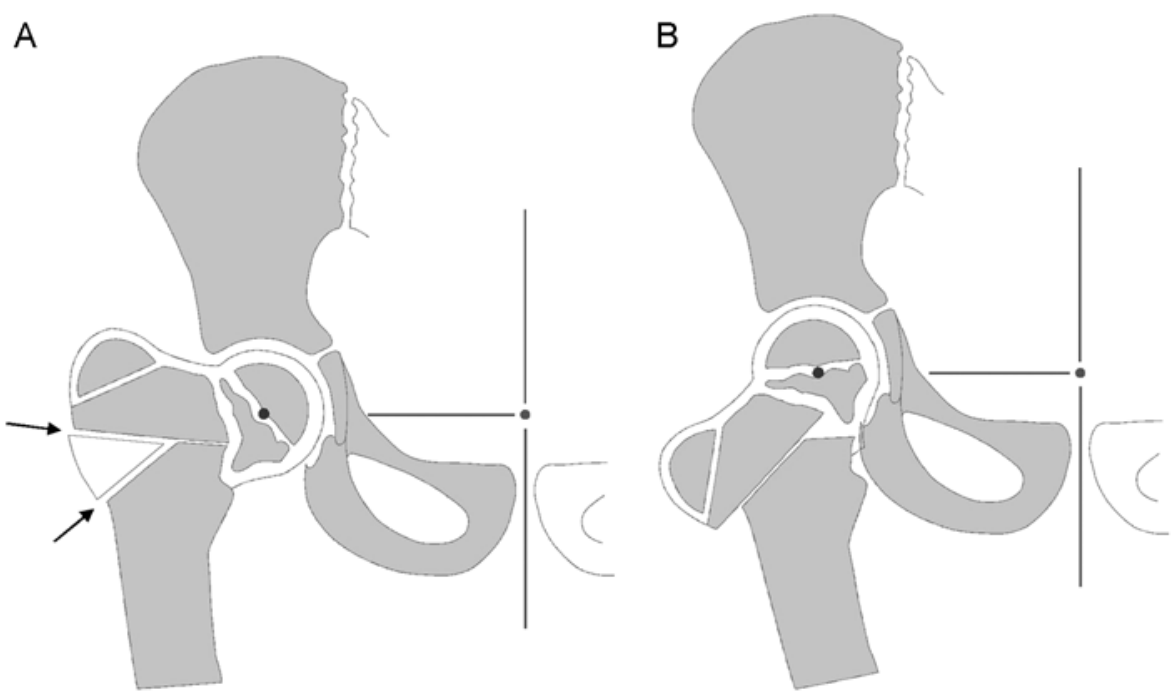

FIGURE 3. A, In Pauwels' procedure, a lateral wedge is extracted using a y-shaped osteotomy. B, Closing the wedge, the femoral neck is valgizated, whereas the femoral shaft is positioned medially. The arrows show the directions of the osteotomies.

have to be altered according to the temperament and circumstances of the individual child. Although physiotherapy is possible for the children without a cast, no weight bearing should be allowed for 6 weeks postoperatively.

\section{Radiologic Analysis}

We analyzed a total of 93 pelvic radiographies with a total of 139 hip joints. Only technically acceptable images were included. Thirty distances, angles, and indices known from the literature ${ }^{21}$ to function as surrogates of the growing hips' morphology, biomechanics, and reactions of the epiphyseal plates to pathological morphologic changes and their biomechanical effects, were assessed.

The following parameters were determined from the pelvic X-rays: the projected angle between the femur's neck and diaphysis (CCD), the angle between Hilgenreiner's line and the epiphyseal plate (EY), the greater trochanter's apophyseal plate angle (AY) as described by Birkenmaier et al, ${ }^{21}$ the articulotrochanteric distance (ATD) as described by Edgren, ${ }^{22}$ Hilgenreiner's AC-angle (AC) and Wiberg's center-edge angle (CE). ${ }^{23}$ Furthermore, the presence of nonunions in the region of the femoral neck was registered (Fig. 6).
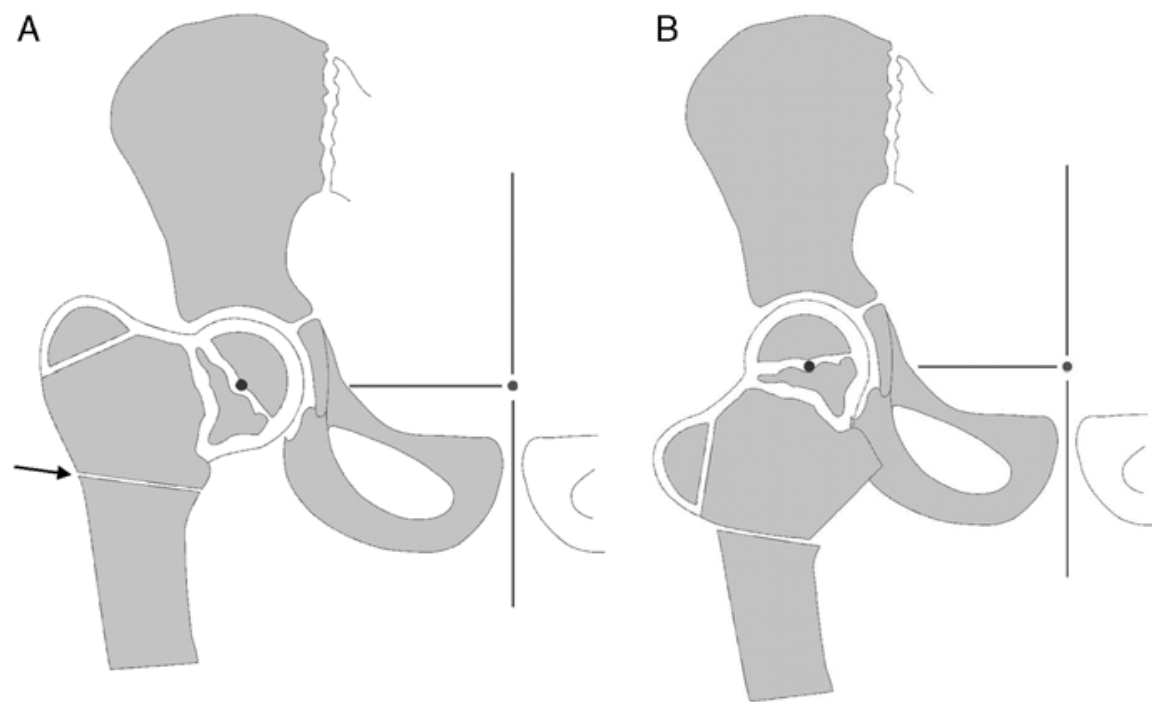

FIGURE 4. A, End-to-side valgization includes a transverse osteotomy below the lesser trochanter without extracting a wedge. B, By abduction of the femur, the lateral cortex of the proximal part is aligned to the osteotomy site. The arrow shows the direction of the osteotomy. 

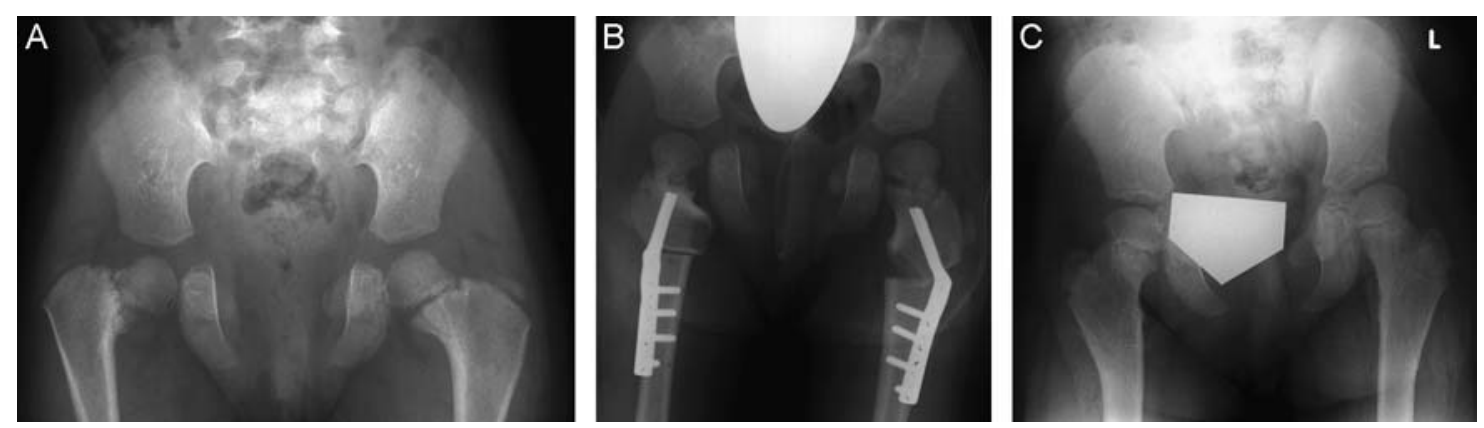

FIGURE 5. A, Preoperative status of a 4-year-old girl with an asymmetric coxa vara congenita. Before the procedure CCD was 85 degrees on the right and 115 degrees on the left side. Right EY was 80 degrees, left EY was 30 degrees. B, On the right hip a subtrochanteric end-to-side valgization osteotomy was performed, whereas on the left hip an intertrochanteric shorthening valgization osteotomy sufficed. C, Follow-up examination 4 years after surgery. CCD, caput collum diaphysis angle; EY, Hilgenreiner-epiphyseal plate angle.

\section{RESULTS}

Preoperatively, 15 out of 20 hips showed nonunions, all of which healed after surgery. We saw one recurrence of deformity at the time of the last follow-up in the only patient with the preoperative diagnosis of congenital coxa vara. Although all of the patients showed Trendelenburg's sign preoperatively, only $2(16.5 \%)$ of them presented one after surgery. Only 1 of 10 patients, who had shown Duchenne's limp before surgery, remained limping postoperatively. There was no sign of preoperative or postoperative partial or complete physeal growth arrest apparent in any of the patients.

\section{CCD Angle}

Preoperatively we assessed a mean collodiaphyseal angle of 98.1 degrees (range, 60 to 128.5 degrees). Two patients presented with CCD angles in excess of 125 degrees, representing the upper limit of coxa vara deform-

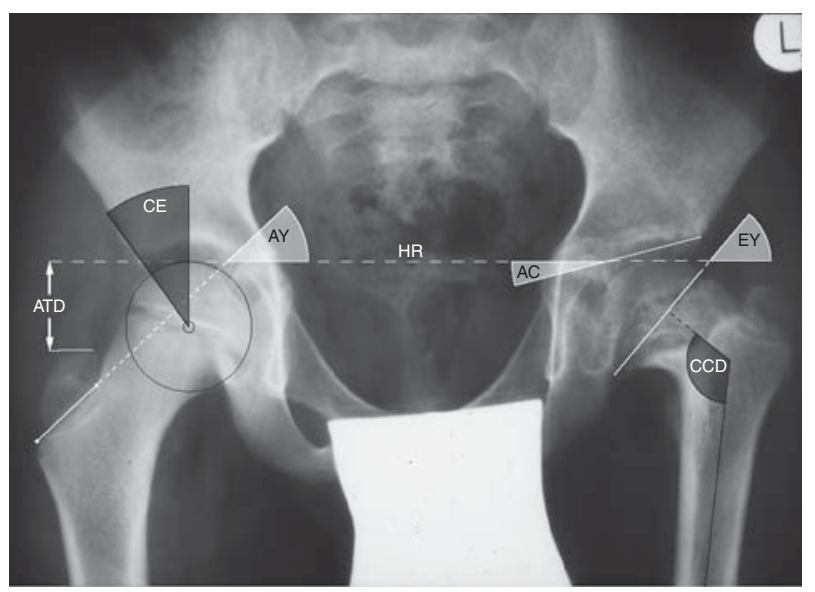

FIGURE 6. Pelvic $x$-ray with an overlay of the described measures. AC indicates Hilgenreinger's acetabular angle; AT, articulotrochanteric distance; AY, Heimkes' apophyseal plate angle; $C C D$, caput collum diaphysis angle; $C E$, Wiberg's center edge angle; EY, Hilgenreiner-epiphyseal plate angle. ities as defined by Fairbank. ${ }^{24}$ Nevertheless, surgery was performed because of a significant aberration from the age appropriate values. Also, in both patients the deformity was the result of an osteomyelitis, which was expected to increase rapidly. Postoperative measurement revealed an average angle of 155.9 degrees (range, 135.0 to 170.0 degrees). Eighty-five months after surgery the mean CCD angle was 143.9 degrees (range, 117.5 to 162.0 degrees). In 1 hip, a varus angle of under 125 degrees could be seen postoperatively. However, that hip showed no additional signs of a coxa vara besides the lowered neck-shaft-angle. The mean femoral neck valgization was 57.8 degrees, reducing to 45.8 degrees $(79.2 \%)$ at 85 months of follow-up (Fig. 7).

\section{EY Angle}

Preoperatively an EY angle of 55.2 degrees (24.5 to 83.0 degrees) was found. After the subtrochanteric osteotomy EY's mean value dropped to 4.9 degrees $(-35.0$ to +46.0 degrees), showing a tendency toward revarization afterwards and reaching an EY angle of a mean $15.7(-2.5$ to +41.5 degrees) at 85 months of follow-up time. In the postoperative course, the negative values increased and an elevated mean value was noted (Fig. 7).

\section{AY Angle}

Preoperatively, a continuous flattening of the trochanteric apophyseal plate could be seen. Although the mean AY angle is 44.5 degrees (26.5 to 59.5 degrees) 30 months before the intervention, it deteriorates due to diminishing abductor force and a steepening trochanteric resultant force vector to 31.8 degrees (6.0 to 56.0 degrees) just before surgery. During surgery, the femoral neck is erected, increasing AY to a mean of 75.0 degrees (38.0 to 97.0 degrees). Postoperatively the ratio between knee extending and hip abducting musculature normalizes, shifting AY toward reference values. Eight-five months after the valgization procedure, a mean value of 66.1 degrees (42.5 to 87.0 degrees) was measured (Fig. 7). 

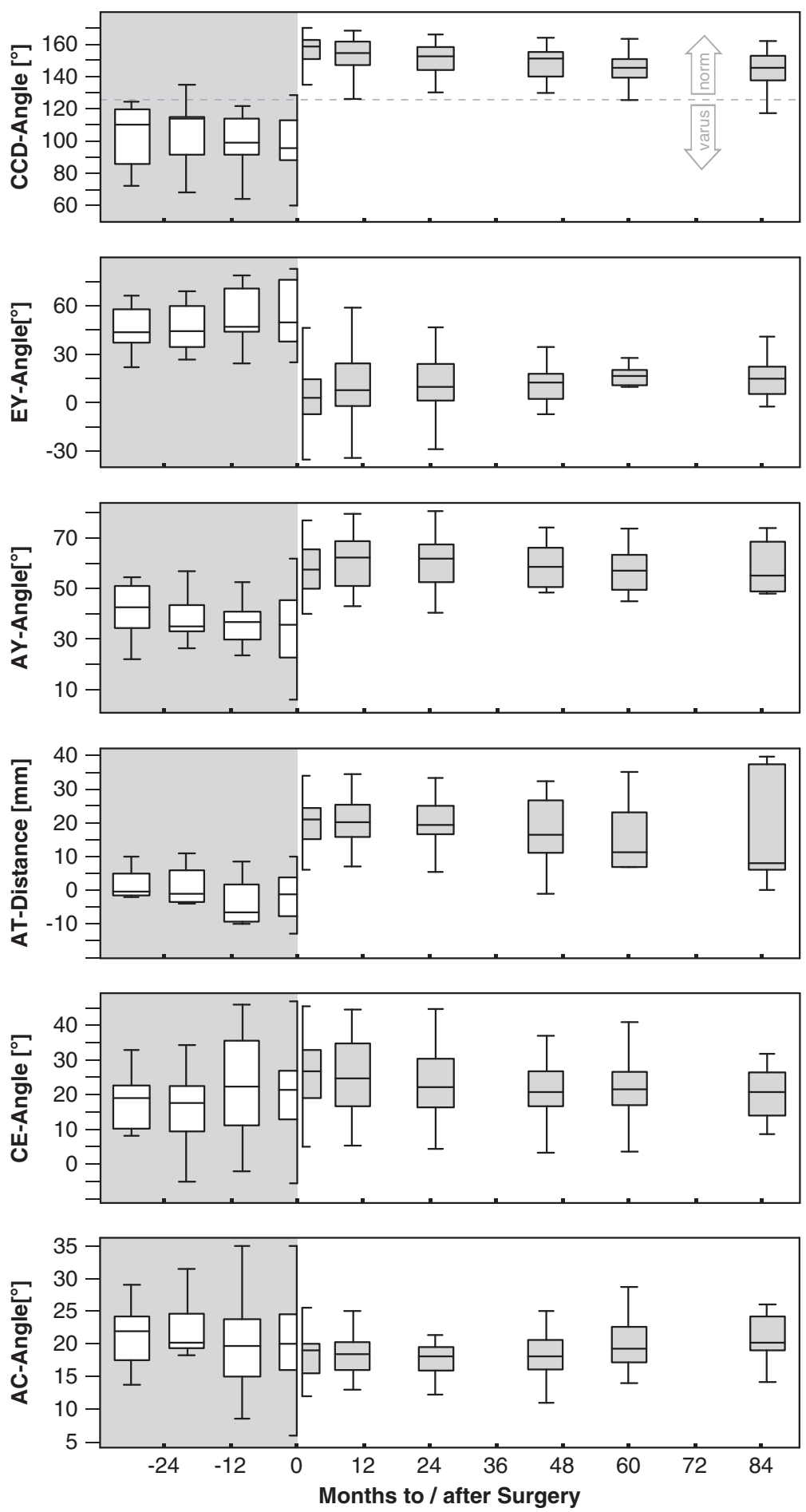

FIGURE 7. Boxplot diagrams displaying the development of CCD, EY AY, CE and AC angles (degrees) as well as the ATD (mm) in relation to the time of surgery (months). The dotted line represents the border between normal and pathologically lowered neckshaft angles as described by Fairbank. ${ }^{24}$ AC indicates Hilgenreinger's acetabular angle; ATD, articulotrochanteric distance; AY, Heimkes' apophyseal plate angle; CCD, caput collum diaphysis angle; CE, Wiberg's center edge angle; EY, Hilgenreinerepiphyseal plate angle.

\section{The ATD}

The ATD immediately before surgery was $-5.3 \mathrm{~mm}$ $(-19.0$ to $+10.0 \mathrm{~mm})$ on average, increasing to $25.7 \mathrm{~mm}$
(15.0 to $39.0 \mathrm{~mm})$ after surgery. At the last follow-up examination, we recorded a mean ATD of $13.7 \mathrm{~mm}$ $(-0.5 \mathrm{~mm}$ to $+40.5 \mathrm{~mm})$, with the decrease resulting from 
a postoperative over-proportional growth of the initially relatively small greater trochanter (Fig. 7).

\section{CE Angle}

Preoperatively we assessed CE angles of 20.4 degrees $(-5.5$ to +47.0 degrees), which increased slightly to 24.5 degrees (5.0 to +45.5 degrees) after surgery. At 85 months after the procedure, the values were lower than the initial measured angles with a mean of 18.4 degrees (8.5 to 32.0 degrees). That means, that after the operation, the femoral head showed increased surrogates of central alignment, dropping to values in the lower areas of the physiological range at 85 months of follow-up (Fig. 7).

\section{AC Angle}

To describe the acetabular slope, the AC angle was determined. Although there was a cut back in the parameter's range, preoperative mean values of 20.2 degrees (6.0 to 35.0 degrees) remained at 20.2 degrees (14.0 to 26.0 degrees). Related to reference values according to age controls, this means a change from initially subnormal to normal and high-range normal values (Fig. 7).

\section{Leg Length}

Unfortunately, preoperative data on leg length were incomplete, rendering analysis of the results of the intervention on the lengthening effect impossible. The mean leg length difference (LLD) after surgery was $2.5 \mathrm{~cm}$ (0 to $15.5 \mathrm{~cm})$. Ten out of 12 patients showed an LLD of $<2.0 \mathrm{~cm}$, whereas the highest LLD of unilaterally operated patients was $1.3 \mathrm{~cm}$. One of the patients underwent an additional ipsilateral lengthening intervention of $4.0 \mathrm{~cm}$ after valgization.

\section{Discussion of Materials and Methods}

The main limitation of the current study is the small number of cases, due to the rarity of the deformity in pediatric patients. As the parameters chosen to assess the surgical outcome vary according to age, it would have been better to divide our sample into several subgroups based on age. However, this would have led to very small numbers of cases within each of the groups, therefore making it impossible to draw valid conclusions.

Additional potential sources of error are the positioning of the patient during the pelvic x-ray and inaccuracies during the measurement procedures.

\section{DISCUSSION}

We hereby present a trial on the subtrochanteric end-to-side valgization osteotomy as described by Borden and colleagues comprising, to the best of our knowledge, the highest number of cases available in the international literature. Other relevant studies were published by Ruszkowski and Kovacic ${ }^{7}(\mathrm{n}=24$, intertrochanteric osteotomy), Weighill and $\operatorname{Orth}^{8}\left(\mathrm{n}_{\text {total }}=32\right.$, intertrochanteric osteotomy $(\mathrm{n}=19)$; subtrochanteric osteotomy $(\mathrm{n}=13)$; various fixation methods), Desai and Johnson ${ }^{9}$ $\left(\mathrm{n}=20\right.$, subtrochanteric osteotomy) and Carroll et al ${ }^{10}$ $\left[\mathrm{n}_{\text {total }}=32\right.$, Pauwel's intertrochanteric osteotomy $(\mathrm{n}=19)$ and subtrochanteric osteotomy $(\mathrm{n}=13)$ ].

Several metric surrogates have been described for hip joints functioning physiologically and pathologically. These parameters showed significant improvement toward reference values, with only slight loss of correction during follow-up examinations.

Nonunions of the femoral neck pose a major complication in coxa vara. Preoperatively, we found pseudarthroses in 15 out of 20 hips $(75 \%)$. After surgery to reduce the shear forces and convert them into compressive forces, all of the nonunions healed without signs of recurrence at the time of the last follow-up examination.

Recurring varization occurred in 2 patients, rendering the relapse rate $10 \%$, which is a low value compared with the studies mentioned above. Although one of the patients had to undergo revision surgery, the other one did not have to be treated, since the nonunion healed in spite of a lowered collodiaphyseal angle.

In the past, the intertrochanteric y-shaped osteotomy of Pauwels was considered state of the art in the therapy of infantile coxa vara, whereas the subtrochanteric end-to-side valgization has not been done or cited frequently. Both techniques are comparable, regarding preload of the hip abductors, the load arm's length, the hip joint's kinematics, and the trochanteric load.

Pauwels's y-shaped osteotomy compromises the hip joint's integrity, potentially endangering the nourishing vessels of the femoral head and thereby includes the risk of an avascular necrosis. In Borden's procedure, there is only minimal risk of head necrosis due to its extra-articular approach. In addition, the end-to-side valgization requires only one osteotomy and low-risk positioning of the angled plate, which makes it technically less demanding in comparison to the intertrochanteric technique.

We did not perform trochanteric apophysiodesis in any of the cases, since in our experience the trochanteric apophysis tends to grow in a lateral rather than a cranial direction after the procedure. In this procedure, the greater trochanter is only partially responsible for loss of correction of the ATD by means of circular growth. At the same time, it contributes to keeping the "trochanteric" portion of the offset steady or even increasing it over time and thus elongating the lever arm of the gluteal muscular system. Of course, valgizating procedures of the proximal femur are stringently associated with a certain amount of secondary postoperative genu valgum. However, none of our patients required axis correcting surgery of the knee.

Both our patients and studies from world literature have shown, that particularly in children with unilateral coxa vara, the deformity is frequently associated with considerable differences of leg length. Moreover, children with bilateral deformities also suffer from the absolute shortening of their lower extremities. Pauwels's osteotomy results in an approximate ipsilateral limb lengthening of 1 diaphyseal diameter, the subtrochanteric 
technique yields a lengthening of 2 diaphyseal diameters. This is a downside to Borden's procedure, which produces some soft tissue tension. Nonetheless, the postoperative resulting flexion-abduction-external rotation contracture of the hip joint is always reversible.

\section{CONCLUSIONS}

The subtrochanteric end-to-side valgization osteotomy is a very effective tool in the therapy of the infantile coxa vara. All of the preoperatively present non-unions healed after the surgery and the rate of revarization was minimal. In our sample, the intervention showed significantly improved hip abductor strength and therefore improved patterns in the observational gait analysis. Furthermore, this method is technically less demanding, safer and more effective regarding the lengthening of the limb. The current trial comprises the highest number of end-to-side valgizations of coxae varae in the literature and compares favorably with existing studies on similar surgical methods.

\section{REFERENCES}

1. Cordes S, Dickens DR, Cole WG. Correction of coxa vara in childhood. The use of Pauwels' Y-shaped osteotomy. J Bone Joint Surg. 1991;73:3-6.

2. Yang SH, Huang SC. Valgus osteotomy for congenital coxa vara. J Formos Med Assoc. 1997;96:36-42.

3. Widmann RF, Hresko MT, Kasser JR, et al. Wagner multiple $\mathrm{K}$-wire osteosynthesis to correct coxa vara in the young child: experience with a versatile "tailor made" high angle blade plate equivalent. J Pediatr Orthop B. 2001;10:43-50.

4. Burns KA, Stevens PM. Coxa vara: another option for fixation. J Pediatr Orthop B. 2001;10:304-310.

5. Sabharval S, Mittal R, Cox G. Percutaneous triplanar femoral osteotomy correction for developmental coxa vara: a new technique. $J$ Pediatr Orthop. 2005;25:28-33.

6. Magu NK, Singh R, Sharma AK, et al. Modified Pauwels intertrochanteric osteotomy in neglected femoral neck fractures in children: a report of 10 cases followed for a minimum of 5 years. J Orthop Trauma. 2007;21:237-243.
7. Ruszkowski I, Kovacic S. Biomechanical analysis of disordered ossification in coxa vara and its normalisation by corrective osteotomies. Arch Orthop Unfallchir. 1973;74:338-344.

8. Weighill FJ, Orth MC. The treatment of the developmental coxa vara by abduction subtrochanteric and intertrochanteric femoral osteotomy with special reference to the role of adductor tenotomy. Clin Orthop Relat Res. 1976;116:116-124.

9. Desai SS, Johnson LO. Long-term results of valgus osteotomy for congenital coxa vara. Clin Orthop Relat Res. 1993;294:204-210.

10. Carroll K, Coleman S, Stevens PM. Coxa vara: surgical outcomes of valgus osteotomies. J Pediatr Orthop. 1997;17:220-224.

11. Magnusson R. Coxa vara infantum. Acta Orthop Scand. 1954; 23:284-308.

12. Johanning K. Coxa vara infantum. I. Clinical appearance and aetiological problems. Acta Othop Scand. 1951;21:273-299.

13. Johanning K. Coxa vara infantum II. Treatment and results of treatment. Acta Orthop Scand. 1952;22:100-125.

14. Amstutz HC, Philip WD. Dysgenesis of the proximal femur (coxa vara) and its surgical management. J Bone Joint Surg Am. 1962;44:1-24.

15. Pauwels F. Biomechanics of the Locomotor Apparatus. Berlin: Springer; 1980.

16. Heimkes B, Posel P, Plitz W, et al. Forces acting on the juvenile hip joint in the one-legged stance. J Pediatr Orthop. 1993;13:431-436.

17. Skuban TP, Vogel T, Baur-Melnyk A, et al. Function-orientated structural analysis of the proximal human femur. Cells Tissues Organs. 2009;190:247-255.

18. Pauwels F. Biomechanics of the Normal and Diseased Hip. Berlin: Springer; 1976.

19. Borden J, Spencer GE, Herndon CH. Treatment of coxa vara in children by means of a modified osteotomy. J Bone Joint Surg. 1966;48A:1106-1110.

20. Heimkes B, Komm M, Melcher C. Subtrochanteric end-to-side valgus osteotomy for severe infantile coxa vara. Oper Orthop Traumatol. 2009;21:97-111.

21. Birkenmaier C, Jorysz G, Jansson V, et al. Normal development of the hip: a geometrical analysis based on planimetric radiography. J Pediatr Orthop B. 2010;19:1-8.

22. Edgren W. Coxa plana. A clinical and radiological investigation with particular reference to the importance of the metaphyseal changes for the final shape of the proximal part of the femur. Acta Orthop Scand. 1965;84(suppl):94-101.

23. Wiberg G. Studies on dysplastic acetabula and congenital dislocation of the hip joint with special reference to the complication of osteoarthritis. Acta Chir Scand. 1939;83(suppl):58.

24. Fairbank HA. Coxa vara due to congenital defect of the neck of the femur. J Anat. 1928;62(pt 2):232-237. 